\title{
Erratum to: Safety and Hemostatic Effectiveness of the Fibrin Pad for Severe Soft-Tissue Bleeding During Abdominal, Retroperitoneal, Pelvic, and Thoracic (Non-cardiac) Surgery: A Randomized, Controlled, Superiority Trial
}

Jonathan Koea,6 ${ }^{1,}$ Peter Baldwin ${ }^{2} \cdot$ Jessica Shen $^{3} \cdot$ B. Patel ${ }^{3} \cdot$ Jonathan Batiller $^{3}$. Axel Arnaud ${ }^{3} \cdot$ James Hart $^{3} \cdot$ Jeffrey Hammond $^{3} \cdot$ Craig Fischer $^{4}$. O. James Garden ${ }^{5}$

Published online: 28 July 2015

(c) Société Internationale de Chirurgie 2015

\section{Erratum to: World J Surg}

DOI 10.1007/s00268-015-3106-5

The first sentence in the Results section of the Abstract is corrected as follows:

At 4 min post-randomization, 50/59 (84.7\%) subjects in the FP group and 10/32 (31.3\%) subjects in the SoC group achieved hemostasis without needing re-treatment $(P<$ 0.0001).

The correct first row entry in Table 3 is Hemostasis at 4 minutes without re-treatment, and the correct standard of treatment for this entry is $10 / 32(31.3 \%)$.

The online version of the original article can be found under doi:10. 1007/s00268-015-3106-5.

Jonathan Koea

jonathan.koea@waitematadhb.govt.nz

1 Department of Surgery, Auckland City Hospital, Grafton,

New Zealand

2 Addenbrooke's Hospital, Cambridge, UK

3 Ethicon, Inc., Somerville, NJ, USA

4 Weill Cornell Medical College of Cornell University, Houston, TX, USA

5 Royal Infirmary of Edinburgh, Edinburgh, UK

6 Department of Surgery, North Shore Hospital, Private Bag 93505, Auckland 0620, New Zealand 